\title{
PRECIFICAÇÃO DA ENERGIA DISPONÍVEL NO BRIQUETE DE CARNAÚBA E CAPIM- ELEFANTE E NA LENHA DO SEMIÁRIDO POTIGUAR
}

\author{
M. A. M. E. TAVARES ${ }^{1}$ e S. R. L. TAVARES ${ }^{2 *}$ \\ ${ }^{1}$ Instituto Federal de Educação, Ciência e Tecnologia do Rio Grande do Norte (IFRN) \\ ${ }^{2}$ Centro Nacional de Pesquisa de Solos - (EMBRAPA) \\ silvio.tavares@embrapa.br
}

Artigo submetido em dezembro/2014 e aceito em agosto/2015

DOI: $10.15628 /$ holos.2015.2661

\section{RESUMO}

Um dos maiores entraves para a consolidação do mercado para briquetes é o mito de que esses biocombustíveis são sempre mais caros do que a lenha advinda do puro extrativismo. Através da análise comparativa do preço da energia disponível para queima em uma tonelada de lenha e briquete misto de palha de carnaúba e capim-elefante, este estudo comprovou que, dependendo do teor de umidade da lenha, o rendimento do briquete é superior em metade das 60 simulações feitas com três tipos de espécies vegetais do semiárido do Rio Grande do Norte. A análise se baseou no cálculo do Poder Calorífico Útil de cada uma das biomassas analisadas e nos preços das respectivas toneladas.

PALAVRAS-CHAVE: Biocombustível sólido, bioenergia, eficiência energética, economia energética, custo energético.

\section{PRICING OF ENERGY AVAILABLE IN CARNAUBA AND ELEPHANT GRASS BRIQUETTE AND POTIGUAR SEMIARID FIREWOOD}

\begin{abstract}
One of the biggest obstacles to market consolidation for briquettes is the myth that these biofuels are always more expensive than pure extraction wood. Through comparative analysis of the price of available energy for burning in a ton of mixed wood and carnauba straw and elephant grass briquette, this study showed that,
\end{abstract}

depending on the wood moisture content, the yield of briquette is superior in half of 60 simulations with three types of plant species in the semiarid region of Rio Grande do Norte. The analysis was based on calculation of Net Calorific Value of each biomass analyzed and the prices of their tons.

KEYWORDS: Solid biofuel, bioenergy, energy efficiency, energy saving, energy cost. 


\section{INTRODUÇÃO}

Os briquetes são uma das formas mais refinadas de biomassa sólida (a outra é o pellet, da qual não trataremos nesse trabalho). São produzidos através da compactação de qualquer matéria orgânica animal e vegetal para fins energéticos. Por oferecerem uma queima rápida e uniforme, são ideais para serem usados em fornos e caldeiras de abatedouros, cerâmicas, cervejarias, destilarias, fecularias, hospitais, hotéis/motéis, indústria de balas, indústria de óleo de soja, indústria de papel, indústria de refrigerantes, laticínios, lavanderias, metalúrgicas, panificadoras, pizzarias, residências e tinturarias (GENTIL, 2008).

No Brasil, na maioria das vezes, os briquetes são vendidos por massa ou por volume e não pela quantidade de energia útil disponível para a queima. Para Gentil (2008), o parâmetro correto na comparação dos preços entre dois biocombustíveis deveria ser a energia efetivamente disponibilizada para ser usada como combustível. Isso é feito através do cálculo do poder calorífico do material.

Assim sendo, o objetivo deste trabalho foi apresentar uma análise de custo-benefício do briquete misto de palha de carnaúba e capim-elefante em relação às espécies lenhosas mais utilizadas na região do Baixo-Açu, que vive hoje um grave processo de desertificação provocado pela retirada insustentável da mata nativa do Bioma Caatinga para queima nos fornos das indústrias cerâmicas locais. Com isso, espera-se estimular a pesquisa de composição de biomassas que ofereçam cada vez mais uma relação custo-benefício interessante para o consumidor, criando mais uma alternativa de combustível, sobretudo nas regiões onde a lenha é escassa ou onde o seu extrativismo tem provocado impactos negativos sobre o meio ambiente.

\section{MATERIAIS E MÉTODOS}

Todos os cálculos e estimativas que permitiram o estabelecimento de preços para a energia efetivamente disponibilizada pelas biomassas estudadas se basearam nos seguintes dados secundários:

- Os dados de Poderes Caloríficos Superior (PCS), Inferior (PCI) e Útil (PCU) do briquete composto $80 \%$ de palha de carnaúba e $20 \%$ de capim-elefante foram obtidos de Tavares \& Santos (2012);

- O PCU da lenha catada foi calculado a partir do PCS que consta no BEN - Balanço Energético Nacional (MINISTÉRIO DAS MINAS E ENERGIA, 2012) para a lenha catada em todo o Brasil de uma forma geral;

- O PCS das duas espécies lenhosas mais utilizadas como lenha no semiárido potiguar, a jurema preta (Mimosa hostilis Benth.) e a algaroba (Prosopis juliflora L) foram estimados por Oliveira et al (1999); Cunha (2012) e Pereira e Lima (2002).

- A correlação entre o metro cúbico estéreo e peso de lenha catada foi extraído de Riegelhaupt (2004);

- O preço do metro cúbico estéreo de lenha legalizada na região do Baixo-Açu foi obtido de Tavares (2013). 


\section{RESULTADOS E DISCUSSÃO}

Os briquetes e pellets são vendidos em unidades de $R \$ / t$ e a lenha em $R \$ / m^{3}$ st, cujo valor é convertido para peso utilizando-se a equação (1), onde $P L$ é o preço da tonelada de lenha; Pst é o preço do metro estéreo dela e $\mathrm{p}$ é a sua densidade:

$$
P L=P s t / P
$$

Já os consumidores precisam da energia da biomassa para gerar calor ou potência em fornalhas, fornos e caldeiras e não simplesmente da sua massa. Quanto mais secas as biomassas, maior é o seu poder calorífico, definido como a "quantidade de energia térmica liberada durante a queima completa de uma unidade de massa ou de volume de combustível, expressa em $\mathrm{kcal} / \mathrm{kg}$, $\mathrm{kJ} / \mathrm{kg}, \mathrm{Kcal} / \mathrm{m}^{3}$ ou $\mathrm{kJ} / \mathrm{m}^{3}$ (TAVARES, 2013). Essa queima é feita em uma câmara adiabática, na qual a amostra é depositada com $0 \%$ de umidade e incinerada, com medições contínuas da temperatura através de uma bomba calorimétrica. A variação da temperatura registrada pela bomba é então utilizada para calcular a energia liberada pelo combustível - chamado de Poder Calorífico Superior (BRAND, 2010), expresso na equação (2), onde $K$ é a constante do calorímetro (cal/ ${ }^{\circ} \mathrm{C}$ ); $\mathrm{mH}_{2} \mathrm{O}$ é o volume da água do calorímetro $(2.700 \mathrm{ml}) ; \mathrm{ms}$ a massa seca da amostra e $\Delta t$ a diferença entre as temperaturas inicial e final da água (ASSOCIAÇÃO BRASILEIRA DE NORMAS TÉCNICAS, 1984).

$$
P C S=\left(K+\mathrm{m} \mathrm{H}_{2} \mathrm{O}\right) \Delta t / m s
$$

Porém, mesmo com teor de umidade da massa de $0 \%$, a reação química provocada pela combustão faz com que as moléculas de hidrogênio constituintes do material se unam às de oxigênio formando água, exigindo um gasto de energia para a sua evaporação. Para se ter um valor mais preciso da quantidade de energia efetivamente disponível no combustível é calculado o PCI ou Poder Calorífico Inferior, que só é igual ao PCS no caso de o material analisado não conter hidrogênio em sua composição. Caso contrário, é excluída a interferência desse vapor d'água produzido durante a combustão no processo de medição do PCS, conforme a equação (3), onde $P C l$ é o Poder Calorífico Inferior (kcal/kg); PCS é o Poder Calorífico Superior ( $\mathrm{Kcal} / \mathrm{Kg}$ ) e $H$ é o percentual de hidrogênio presente no material (BRAND, 2010).

$$
P C l=P C S-600 \times 9 H / 100
$$

O PCl, por sua vez, só pode ser usado diretamente como parâmetro se o combustível não apresentar umidade livre, externa. Caso contrário, segundo Brand (2010), deve-se utilizar para o cálculo de energia disponível o Poder Calorífico Útil (PCU), expresso na equação (4), na qual o $P C U$ = Poder Calorífico Útil; PCS = Poder Calorífico Superior; $K=$ constante de calor de vaporização da água no calorímetro, no valor de $324 \mathrm{kcal} / \mathrm{kg} ; 600$ = constante; 238,89 = transformação de $\mathrm{kcal} / \mathrm{kg}$ em MJ/Kg ou GJ/t e TU = teor de umidade (GENTIL, 2008).

$$
P C U=[(P C S-K) *(1-T U)-(600 * T U)] / 238,89
$$

A razão entre o preço da lenha (equação 1) e o seu Poder Calorífico Útil (equação 4) resulta no preço da energia útil (PE) do biocombustível, expresso em $R \$$ / GJ ou em $R \$$ /MJ, conforma a equação 5 (GENTIL, 2008):

$$
P E=P L / P C U
$$


A lenha legalizada ${ }^{1}$ é vendida a um preço médio de $\mathrm{R} \$ 35,00$ o $\mathrm{m}^{3}$ st (TAVARES, 2013); já para o briquete misto foram estabelecidos quatro cenários de preço para a tonelada: $\mathrm{R} \$ 250,00$, $\mathrm{R} \$ 275,00, \mathrm{R} \$ 300,00, \mathrm{R} \$ 325,00$ e $\mathrm{R} \$ 350,00$. Para permitir uma melhor comparação entre os gastos com lenha e com briquete será usada inicialmente a unidade de massa (tonelada) como unidade para os dois combustíveis. Sendo assim,

- $1 \mathrm{~m}^{3}$ st de lenha =0,21 t (RIEGELHAUPT, 2004). Logo,

- $1 \mathrm{t}=4,7 \& 6 \mathrm{~m}^{3}$ st de lenha $=4,76 \times \mathrm{R} \$ 35,00=\mathrm{R} \$ 166,67$ (custo médio de 1 tonelada de lenha legal da caatinga na região do Baixo-Açu (2013);

$\mathrm{Na}$ comparação entre dois combustíveis, é necessário calcular o poder energético de ambos:

- $\quad$ PCS da lenha catada $=3.300 \mathrm{kcal} / \mathrm{kg}$ (MINISTÉRIO DAS MINAS E ENERGIA,2012).

- $\quad$ PCS da lenha de jurema preta $=4.150 \mathrm{kcal} / \mathrm{kg}$ (OLIVEIRA et al, 1999; CUNHA, 2012);

- $\quad$ PCS da lenha de algaroba $=4.935 \mathrm{kcal} / \mathrm{kg}$ (OLIVEIRA et al, 1999; PEREIRA \& LIMA, 2002; CUNHA, 2012)

- PCS do briquete composto $80 \%$ de palha de carnaúba e $20 \%$ de capim elefante $=4.318$ $\mathrm{kcal} /$ KG (TAVARES E SANTOS, 2012);

- PCS do briquete composto $100 \%$ de palha de carnaúba = $4.395 \mathrm{kcal} / \mathrm{KG}$ (SANTOS, 2012);

Aplicando-se a fórmula (3), obtivemos os seguintes valores para os Poderes Caloríficos Inferiores dos materiais testados:

- $\quad \mathrm{PCl}$ da lenha catada $=2.976 \mathrm{kcal} / \mathrm{kg}$;

- $\mathrm{PCl}$ da lenha de jurema $=3.826 \mathrm{kcal} / \mathrm{kg}$;

- $\quad \mathrm{PCl}$ da lenha de algaroba $=4.611 \mathrm{kcal} / \mathrm{kg}$;

- $\mathrm{PCl}$ do briquete composto $100 \%$ de palha de carnaúba $=4.092 \mathrm{kcal} / \mathrm{kg}$

- $\mathrm{PCl}$ do briquete composto $80 \%$ de palha de carnaúba e $20 \%$ de capim elefante $=3.994$ $\mathrm{kcal} /$ KG.

Conforme os dados acima indicam, o $\mathrm{PCl}$ do briquete com $100 \%$ de carnaúba e $80 \%$ de carnaúba $+20 \%$ de capim-elefante são $35 \%$ em média maiores, respectivamente, do que o $\mathrm{PCl}$ da lenha catada, obtido através do PCS definido pelo BEN. Ou seja: para se ter a mesma quantidade de energia contida em 1 tonelada de briquete de palha de carnaúba é necessário adquirir $35 \%$ a mais de lenha em massa. No caso dos consumidores de lenha legal do Baixo-Açu, que compram a tonelada de lenha legal por $\mathrm{R} \$ 166,67$, o gasto para obter, com este combustível, a mesma quantidade de energia que teriam com briquete seria de $\mathrm{R} \$ 166,67+35 \%=\mathbf{R} \mathbf{\mathbf { 2 2 2 5 } , 0 0}$.

\footnotetext{
${ }^{1}$ Nesse trabalho utilizou-se como termo de comparação ao briquete misto de palha de carnaúba e capim-elefante o preço da lenha legal, já que não se pode comparar os preços de venda da lenha ilegal, que não possui outro custo de produção a não ser a sua coleta e o transporte, uma vez que na região, a comercialização de lenha se trata de uma atividade normalmente ilegal.
} 
Já o $\mathrm{PCl}$ da lenha de jurema preta é apenas 4,39\% inferior do que o do briquete misto, 0 que faz com que ela tenha um rendimento maior do que a lenha catada de $\mathrm{R} \$ 166,67+4,39 \%=\mathbf{R} \$$ 174,00 .

No caso da lenha da algaroba, cujo $\mathrm{PCl}$ é $15,45 \%$ superior ao do briquete, o preço da respectiva tonelada de energia é de $\mathrm{R} \$ 166,67-15,45 \%=\mathbf{R} \mathbf{\$} \mathbf{1 4 1 , 0 0}$, bem inferior aos das demais biomassas.

O PCl serve apenas de ponto de partida para se estabelecer o rendimento de uma biomassa em relação a outra, pressupondo-se que elas tenham teor de umidade externa igual a zero, o que não acontece na natureza. A Tabela 1 mostra o preço relativo dessas biomassas, considerando os seus Poderes Caloríficos Úteis, que variam conforme os teores de umidade externa e que foram calculados a partir dos PCS citados anteriormente. Já o PCU do briquete é fixo e foi calculado em $3.627 \mathrm{kcal} / \mathrm{kg}$, de acordo com o PCS definido por Tavares e Santos (2012). O que varia são os preços da tonelada do produto estabelecidos para diferentes cenários do mercado para briquetes no semiárido potiguar.

Já a Tabela 2 expõe as diferentes relações de preço da energia efetivamente disponibilizada pelas quatro biomassas analisadas, levando-se em conta as cinco faixas de preços estabelecidos para o briquete misto no Vale do Açu. As anotações em vermelho expressam a vantagem do briquete em relação aos três diferentes tipos de lenha.

Tabela 1. Preços da energia oriunda da lenha e do briquete na região do Baixo-Açu

\begin{tabular}{|c|c|c|c|c|c|}
\hline $\begin{array}{c}\text { UMID. } \\
\%\end{array}$ & $\begin{array}{c}\text { LENHA CATADA } \\
\mathrm{R} \$ 225,00 / \mathrm{t}\end{array}$ & $\begin{array}{c}\text { JUREMA PRETA } \\
\mathrm{R} \$ 174,00 / \mathrm{t} \\
\end{array}$ & $\begin{array}{l}\text { ALGAROBA } \\
\mathrm{R} \$ 141,00 / \mathrm{t} \\
\end{array}$ & $\begin{array}{c}\text { BRIQUETE } \\
\mathrm{R} \$ / \mathrm{t}\end{array}$ & $\begin{array}{c}\text { BRIQUETE } \\
\text { PE (R\$/kcal) }\end{array}$ \\
\hline \multirow{5}{*}{0} & \multirow{5}{*}{$\begin{array}{c}\text { PCU } \\
2.976 \mathrm{kcal} / \mathrm{kg} \\
\mathrm{PE} \\
\mathrm{R} \$ 0,0000756 / \mathrm{Kcal}\end{array}$} & \multirow{5}{*}{$\begin{array}{c}\text { PCU } \\
3.826 \mathrm{kcal} / \mathrm{kg} \\
\mathrm{PE} \\
\mathrm{R} \$ 0,0000455 / \mathrm{Kcal}\end{array}$} & \multirow{5}{*}{$\begin{array}{c}\text { PCU } \\
4.611 \mathrm{kcal} / \mathrm{kg} \\
\mathrm{PE} \\
\mathrm{R} \$ 0,0000306 / \mathrm{Kcal}\end{array}$} & 250,00 & 0,0000689 \\
\hline & & & & 275,00 & 0,0000758 \\
\hline & & & & 300,00 & 0,0000827 \\
\hline & & & & 325,00 & 0,0000896 \\
\hline & & & & 350,00 & 0,0000965 \\
\hline \multirow{5}{*}{10} & \multirow{5}{*}{$\begin{array}{c}\text { PCU } \\
2.941 \mathrm{Kcal} / \mathrm{kg} \\
\mathrm{PE} \\
\mathrm{R} \$ 0,0000859 / \mathrm{Kcal}\end{array}$} & \multirow{5}{*}{$\begin{array}{c}\text { PCU } \\
3.383 \mathrm{Kcal} / \mathrm{kg} \\
\mathrm{PE} \\
\mathrm{R} \$ 0,0000514 / \mathrm{Kcal}\end{array}$} & \multirow{5}{*}{$\begin{array}{c}\text { PCU } \\
4.611 \mathrm{kcal} / \mathrm{kg} \\
\mathrm{PE} \\
\mathrm{R} \$ 0,0000306 / \mathrm{Kcal}\end{array}$} & 250,00 & 0,0000689 \\
\hline & & & & 275,00 & 0,0000758 \\
\hline & & & & 300,00 & 0,0000827 \\
\hline & & & & 325,00 & 0,0000896 \\
\hline & & & & 350,00 & 0,0000965 \\
\hline \multirow{5}{*}{20} & \multirow{5}{*}{$\begin{array}{c}\text { PCU } \\
2.261 \mathrm{Kcal} / \mathrm{kg} \\
\mathrm{PE} \\
\mathrm{R} \$ 0,0000995 / \mathrm{Kcal}\end{array}$} & \multirow{5}{*}{$\begin{array}{c}\mathrm{PCU} \\
2.941 \mathrm{Kcal} / \mathrm{kg} \\
\mathrm{PE} \\
\mathrm{R} \$ 0,0000592 / \mathrm{Kcal}\end{array}$} & \multirow{5}{*}{$\begin{array}{c}\text { PCU } \\
3.569 \mathrm{Kcal} / \mathrm{kg} \\
\mathrm{PE} \\
\mathrm{R} \$ 0,0000395 / \mathrm{Kcal}\end{array}$} & 250,00 & 0,0000689 \\
\hline & & & & 275,00 & 0,0000758 \\
\hline & & & & 300,00 & 0,0000827 \\
\hline & & & & 325,00 & 0,0000896 \\
\hline & & & & 350,00 & 0,0000965 \\
\hline \multirow{5}{*}{30} & \multirow{5}{*}{$\begin{array}{c}\mathrm{PCU} 1.903 \mathrm{Kcal} / \mathrm{kg} \\
\mathrm{PE} \\
\mathrm{R} \$ 0,0001182 / \mathrm{Kcal}\end{array}$} & \multirow{5}{*}{$\begin{array}{c}\text { PCU } \\
2.498 \mathrm{Kcal} / \mathrm{kg} \\
\mathrm{PE} \\
\mathrm{R} \$ 0,0000697 / \mathrm{Kcal}\end{array}$} & \multirow{5}{*}{$\begin{array}{c}\text { PCU } \\
3.048 \mathrm{Kcal} / \mathrm{kg} \\
\mathrm{PE} \\
\mathrm{R} \$ 0,0000463 / \mathrm{Kcal}\end{array}$} & 250,00 & 0,0000689 \\
\hline & & & & 275,00 & 0,0000758 \\
\hline & & & & 300,00 & 0,0000827 \\
\hline & & & & 325,00 & 0,0000896 \\
\hline & & & & 350,00 & 0,0000965 \\
\hline \multirow{5}{*}{40} & \multirow{5}{*}{$\begin{array}{c}\text { PCU } \\
1.546 \mathrm{kcal} / \mathrm{kg} \\
\mathrm{PE} \\
\mathrm{R} \$ 0,0001455 / \mathrm{kcal}\end{array}$} & \multirow{5}{*}{$\begin{array}{c}\text { PCU } \\
2.056 \mathrm{kcal} / \mathrm{kg} \\
\mathrm{PE} \\
\mathrm{R} \$ 0,0000846 / \mathrm{kcal}\end{array}$} & \multirow{5}{*}{$\begin{array}{c}\text { PCU } \\
2.527 \mathrm{kcal} / \mathrm{kg}) \\
\mathrm{PE} \\
\mathrm{R} \$ 0,0000558 / \mathrm{kcal}\end{array}$} & 250,00 & 0,0000689 \\
\hline & & & & 275,00 & 0,0000758 \\
\hline & & & & 300,00 & 0,0000827 \\
\hline & & & & 325,00 & 0,0000896 \\
\hline & & & & 350,00 & 0,0000965 \\
\hline \multirow{5}{*}{50} & \multirow{5}{*}{$\begin{array}{c}\text { PCU } \\
1.188 \mathrm{kcal} / \mathrm{kg} \\
\text { PE } \\
\mathrm{R} \$ 0,0001894\end{array}$} & \multirow{5}{*}{$\begin{array}{c}\text { PCU } \\
1.613 \mathrm{kcal} / \mathrm{kg} \\
\text { PE } \\
\mathrm{R} \$ 0,0001079\end{array}$} & \multirow{5}{*}{$\begin{array}{c}\mathrm{PCU} \\
2.527 \mathrm{kcal} / \mathrm{kg} \\
\mathrm{PE} \\
\mathrm{R} \$ 0,0000558 / \mathrm{kcal}\end{array}$} & 250,00 & 0,0000689 \\
\hline & & & & 275,00 & 0,0000758 \\
\hline & & & & 300,00 & 0,0000827 \\
\hline & & & & 325,00 & 0,0000896 \\
\hline & & & & 350,00 & 0,0000965 \\
\hline
\end{tabular}


Tabela 2. Relação preço do briquete / preços dos três tipos de lenhas de acordo a umidade livre

\begin{tabular}{|c|c|c|c|c|c|c|c|c|}
\hline \multirow{2}{*}{ Espécies de lenha } & \multirow{2}{*}{$\begin{array}{c}\text { Preço do } \\
\text { briquete* } \\
\text { (R\$/t) }\end{array}$} & \multirow{2}{*}{$\begin{array}{l}\text { Preço da } \\
\text { energia } \\
\text { do } \\
\text { briquete }\end{array}$} & \multicolumn{6}{|c|}{ Teores de umidade dos diferentes tipos de lenha } \\
\hline & & & $0 \%$ & $10 \%$ & $20 \%$ & $30 \%$ & $40 \%$ & $50 \%$ \\
\hline \multirow{5}{*}{$\begin{array}{l}\text { Lenha catada } \\
\text { (R\$ 225,00/t) }\end{array}$} & 250,00 & 0,0000689 & 8,83 & 19,80 & 30,74 & 41,70 & 52,64 & 63,61 \\
\hline & 275,00 & 0,0000758 & 0,28 & 11,78 & 23,81 & 35,87 & 47,90 & 59,97 \\
\hline & 300,00 & 0,0000827 & 9,40 & 3,76 & 16,88 & 30,04 & 43,17 & 56,33 \\
\hline & 325,00 & 0,0000896 & 18,52 & 4,26 & 9,96 & 24,21 & 38,43 & 52,69 \\
\hline & 350,00 & 0,0000965 & 27,64 & 12,28 & 3,03 & 18,38 & 33,69 & 49,05 \\
\hline \multirow{5}{*}{$\begin{array}{l}\text { Jurema preta } \\
\text { (R\$ 174,00/t) }\end{array}$} & 250,00 & 0,0000689 & 51,56 & 34,01 & 16,50 & 1,05 & 18,55 & 36,10 \\
\hline & 275,00 & 0,0000758 & 66,72 & 47,41 & 28,15 & 8,85 & 10,41 & 29,71 \\
\hline & 300,00 & 0,0000827 & 81,87 & 60,81 & 39,80 & 18,75 & 2,27 & 23,32 \\
\hline & 325,00 & 0,0000896 & 97,03 & 74,22 & 51,45 & 28,64 & 5,88 & 16,93 \\
\hline & 350,00 & 0,0000965 & 112,19 & 87,62 & 63,10 & 38,54 & 14,02 & 10,54 \\
\hline \multirow{5}{*}{$\begin{array}{c}\text { Algaroba } \\
(\mathrm{R} \$ 141,00 / t)\end{array}$} & 250,00 & 0,0000689 & 125,41 & 99,94 & 74,47 & 49,00 & 23,53 & 1,94 \\
\hline & 275,00 & 0,0000758 & 147,95 & 119,93 & 91,92 & 63,90 & 35,88 & 7,87 \\
\hline & 300,00 & 0,0000827 & 170,49 & 139,93 & 109,36 & 78,80 & 48,24 & 17,68 \\
\hline & 325,00 & 0,0000896 & 193,03 & 159,92 & 126,81 & 93,70 & 60,59 & 27,48 \\
\hline & 350,00 & 0,0000965 & 215,57 & 179,91 & 144,26 & 108,60 & 72,94 & 37,29 \\
\hline
\end{tabular}

\section{CONCLUSÃO}

A análise da Tabela 2 nos permite chegar à conclusão que, diante de um PCU tão alto como o da algaroba, mesmo com teores de umidades de $40 \%$, considerada alta para uma biomassa que será usada na geração de energia térmica, torna-se muito difícil sua substituição pela lenha artificial, uma vez que a própria natureza dotou esse vegetal das características que a tecnologia tenta acrescentar às biomassas artificiais, como é o caso do briquete. Contudo, há que se considerar que, como no Rio Grande do Norte não existem florestas energéticas de algaroba, o fato de a lenha dessa árvore apresentar uma relação custo-benefício excepcional não significa que hoje seja possível atender, apenas com ela, a uma parcela considerável da demanda por lenha e carvão vegetal.

Em relação à jurema preta, a partir de uma umidade de 30\%, o uso do briquete misto pode ser mais vantajoso, se o preço da tonelada for inferior a $\mathrm{R} \$ 275,00$. Com uma umidade de $40 \%$, ainda que o briquete custe $\mathrm{R} \$ 300,00$ ele representará economia para o consumidor.

Já a lenha genérica, formada por galhos de fruteiras, restos de podas urbanas, entre outras biomassas presentes em todo o País, inclusive no semiárido nordestino, mesmo com umidade zero se mostrou menos vantajosa que o briquete mais barato; a partir de $10 \%$ de umidade o briquete passa a ser mais vantajoso na maior parte das simulações.

Enfim, se considerarmos que não existe lenha disponibilizada na natureza com zero por cento de umidade e que o teor médio de umidade do material lenhoso se situa acima dos $20 \%$ ( $25 \%$ de acordo com o BEN), pode-se observar que a partir dessa faixa, metade das 60 simulações se mostraram favoráveis ao uso do briquete.

É importante destacar que esse estudo foi proveniente de uma simulação real aplicada em uma fábrica de briquetes (Briquete Vale do Açu), localizada no município de Ipanguaçu,-RN, em um cenário de produção de briquetes o mais conservador possível, para a elaboração do plano de 
negócios da fábrica. Neste cenário, utiliza-se como reserva estratégica a mistura de $20 \%$ de biomassa de capim-elefante plantado sob irrigação, com um custo fixo de $R \$$ 80,00 (EMBRAPA, 2013) a tonelada.

Estudos realizados na região (TAVARES, 2013) estimam que a oferta de palha de carnaúba no Baixo-Açu seja suficiente para abastecer 5 fábricas como a citada, com capacidade de produzir 4.800 t/ano, a um preço médio de $\mathrm{R} \$ 25,00$ a tonelada da matéria-prima. Comparando com o preço médio da tonelada de biomassa deste trabalho $(R \$ 50,40)$, pode-se inferir que essa diferença de custo $(R \$ 25,40)$, poderia ser subtraída do preço de venda da tonelada do briquete, colocando-

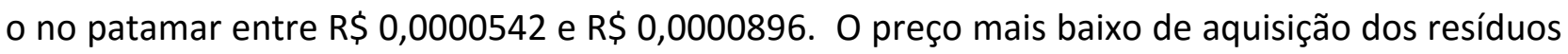
da carnaúba alteraria substancialmente o cenário geral do preço da energia do briquete, tornando ainda mais vantajosa a utilização desse biocombustível sólido, comparativamente à lenha.

\section{REFERÊNCIAS BIBLIOGRÁFICAS}

1. ASSOCIAÇÃO BRASILEIRA DE NORMAS TÉCNICAS. NBR 8633: determinação do poder calorífico superior, NBR 8633. [Rio de Janeiro],1984.

2. BRAND. M. A. Energia de biomassa florestal. Rio de Janeiro: Interciência, 2010.

3. MINISTÉRIO DE MINAS E ENERGIA. Balanço energético nacional 2012: ano base 2011. Rio de Janeiro: Empresa de Pesquisa Energética, 2012. Disponível em: <https://ben.epe.gov.br/>. Acesso em: 15 jul. 2012.

4. CUNHA, A. B. Análise das propriedades físicas, mecânicas e energéticas da parte aérea e tronco de algaroba (Prosopis Juliflora). 2012. 40 f. Trabalho de Conclusão de Curso (Engenharia Florestal) - Universidade de Brasília, Brasília. Disponível em $<$ http://bdm.bce.unb.br/bitstream/10483/4438/1/2012_AndreBarretoCunha.pdf>. Acesso em 10 nov. 2012.

5. GENTIL, L. V. B. Tecnologia e economia do briquete de madeira. 2008. 197f. Tese (Doutorado em Engenharia Florestal) - Universidade de Brasília, Brasília.

6. OLIVEIRA, M. R. de et al. Estudo das condições de cultivo da algaroba e jurema preta e determinação do PC. Revista de Ciência \& Tecnologia, São Paulo, v. 14, p. 93-104, 1999.

7. PEREIRA, J. C. D; LIMA, P. C. F. Comparação da qualidade da madeira de seis espécies de algorobeira para a produção de energia. Colombo: Embrapa Florestas, 2002. (Embrapa Florestas. Boletim de Pesquisa e Desenvolvimento, 45).

8. RIEGELHAUPT, E. Revisão e atualização da oferta e demanda de energéticos florestais no Nordeste. Brasília, 2004. Relatório Final do Consultor - Projeto TCP/BRA/2909.

9. TAVARES, M.A.M.E. Estudo da viabilidade da produção de briquete e seus possíveis impactos sobre o meio ambiente e o mercado de trabalho da região do Baixo-Açu, RN. 2013. $245 \mathrm{f}$. Dissertação (Mestrado em Economia) - Universidade Federal da Paraíba, João Pessoa, PB.

10. TAVARES, S. R. de L. \& SANTOS, T. E. dos. Potencial de uso de biomassa vegetal para a produção de briquetes na região do baixo-açu no Rio Grande do Norte. In: CONGRESSO INTERNACIONAL DE BIOENERGIA, 7; BIOTECH FAIR, 5. 2012, São Paulo. 